\title{
De la imposibilidad de un mosaicismo infértil en yegua criolla colombiana hacia la restauración de la red hormonal reproductiva, reporte de caso
}

\author{
ANA CATALINA DUARTE SANCHEZ ${ }^{1}$ \\ Corresponding Author: ANA CATALINA DUARTE SANCHEZ, catydusa@yahoo.com \\ ${ }^{1}$ Medica Veterinaria y Zootecnista | catydusa@yahoo.com
}

\begin{abstract}
El mosaicismo es uno de los problemas citogeneticos mas frecuentes en yeguas, de difícil diagnostico, ya que las yeguas no presentan un patrón fenotípico especifico, en donde es poco probable o improbable la reproducción de los animales que lo padecen. Esto trae como consecuencias, perdidas económicas de gran impacto a los criadores de caballos; por ser descartadas por su inactividad reproductiva. Los tratamientos de tipo hormonal son usados por algunos médicos veterinarios para intentar que estos animales tengan un ciclo estral normal. En el paciente del reporte del caso en mención, se realizaron protocolos hormonales con resultados insatisfactorios. Posteriormente se realizo un examen citogenetico, dado que se había descartado problemas infecciosos o inflamatorios. Se diagnostica como yegua mosaico, problemas de infertilidad, considerándose finalizada su actividad reproductiva. Se realizo un tratamiento con terapia bioreguladora, logrando estimular el sistema hormonal y celular del paciente y obteniendo resultados positivos. En la actualidad se han obtenido 4 embriones viables los cuales fueron transferidos en receptoras, $\mathrm{y}$ de estos cuatro embriones dos fueron reabsorbidos por las yeguas receptoras, y los otros dos embriones gestados, actualmente son potros con de 16 y 15 meses de edad respectivamente.

Este caso, nos da una luz para integrar la medicina bioreguladora en todos los aspectos que afecten a nuestros pacientes y nos muestra un nuevo aspecto en cuanto a avances a nivel reproductivo en animales.
\end{abstract}

Keywords: mosaicismo en caballos, reporte de caso, examen citogenetico, altrenogest, infertilidad

\section{$\underline{\text { Introduction }}$}

La infertilidad en las especies se define como la incapacidad de concebir o llevar a término una gestación; esta puede ser de origen multifactorial, ya sea por infecciones (bacterias, hongos, virus), inflamación crónica, traumas, o factores genéticos.

La presencia de anomalías cromosómicas en equinos como el mosaico 63,XO/64,XX (presencia de dos o más tipos de líneas celulares presentes en un organismo, de origen pre-cigótico o pos-cigótico) 
disminuye el desempeño reproductivo de yeguas fenotipicamente normales porque afecta el desarrollo del tracto reproductivo: ovarios muy pequeños y/o quísticos, cuerpos lúteos persistentes, atrofia endometrial y/o flacidez cervical; lo que se hace evidente por la presentación de ciclos irregulares, anovulatorios o anestros prolongados $(1,2,3)$.El pronostico de este tipo de pacientes es malo, son muy pocas o ninguna las probabilidades de que se puedan reproducir. El mosaicismo 64,XX/63,XO en yeguas es la tercera anomalía cromosómicas más frecuente después del síndrome de Turner o monosomía del X $(63, \mathrm{XO})$ y el Síndrome de Sexo Reverso o disgenesia gonadal $(64, \mathrm{XY})(4)$. Las terapias convencionales para estos problemas reproductivos son reducidas y en ocasiones inexistentes, dejando al clínico sin herramientas para este tipo de pacientes, recurriendo a terapias hormonales, como los estrogenos, progestagenos, prostaglandinas, con altos porcentajes de fallas en los resultados. El valor genético del paciente, y la trayectoria competitiva de su ascendencia, crea un reto clínico para la elección y tratamiento con medicamentos bioreguladores. El caso de este paciente es especial ya que este tipo de terapias no se reportan que se hayan realizado en equinos con algun resultado de exito.

\section{$\underline{\text { Timeline }}$}

2018-03-08 - $\begin{aligned} & \text { Yegua con problemas reproductivos, no presenta ciclos estrales. examen con } \\ & \text { ultrasonido vía rectal, el útero tamaño de } 3 \mathrm{~cm} \text { de diámetro, ovarios de } 2,0 \mathrm{~cm} \text { de } \\ & \text { diámetro sin ninguna estructura importante.realizan protocolo hormonal con } \\ & \text { altrenogest }\end{aligned}$
$\begin{gathered}\text { 2018-03-26 — } \\ \text { Palpación rectal con ultrasonido, no hay cambio de las estructuras ováricas, ovarios } \\ \text { de diámetro 2,0 cm, y útero de diámetro } 3,0 \mathrm{~cm} \text {.(tratamiento y examenes del anterior } \\ \text { veterinario) }\end{gathered}$
$\begin{aligned} & \text { Palpación rectal con ultrasonido, se encuentran los ovarios con estructuras foliculares } \\ & \text { en crecimiento y grado } 2 \text { de edema en el útero (tratamiento y exámenes del anterior } \\ & \text { veterinario) }\end{aligned}$

2018-04-23 - Yegua inseminada 8 días antes.La yegua es sometida al lavado uterino y no arroja ninguna estructura embrionaria.(tratamiento y exámenes del anterior veterinario) irregularidad de tiempo entre uno y otro, fue inseminada en repetidas ocasiones, sin ningún resultado de embrion o de preñez .(reporte del medico veterinario anterior) 
2019-01-25 - Se toma muestra de sangre en un tubo con heparina y se envía al laboratorio de la Universidad Nacional de Colombia, a la unidad de citogenética y de genotipificación de animales domésticos.

Resultado de laboratorio: Ejemplar mosaico con dos lineas celulares:63,X0 /64,XX. Se encuentran celulas con numero cromosomico anormal 63,X0(42\%) y celulas con numero cromosomico normal 64,XX (58\%)Yegua con problemas reproductivos, yegua mosaico,infertil pequeño plano, ovarios sin ninguna estructura ovárica visible.

Se inicia tratamiento biorregulador con Nuxeel Homaccord ad.us.vet, Berbeel Homaccord ad.us.vet, Lymphomyosot N, Hormeel SN, Hypophysis suis - Injeel, Ovarium compositum ad.us.vet Coenzyme compositum ad.us.vet

Segunda sueroterapia intravemosa. En un 1 litro de cloruro de sodio 1 ampolla de Nuxeel Homaccord ad.us.vet, Berbeel Homaccord ad.us.vet, Lymphomyosot N, Hormeel SN, Hypophysis suis - Injeel, Ovarium compositum ad.us.vet Coenzyme compositum ad.us.vet

Se realiza tercera sueroterapia bioreguladora.Palpación rectal con ultrasonido, se observa el utero de un diametro de 3,5cm y los ovarios de $2,5 \mathrm{~cm}$ aproximadamente

Cuarta sueroterapia intravemosa. En un 1 litro de cloruro de sodio 1 ampolla de Nuxeel Homaccord ad.us.vet, Berbeel Homaccord ad.us.vet, Lymphomyosot N, Hormeel SN, Hypophysis suis - Injeel, Ovarium compositum ad.us.vet Coenzyme compositum ad.us.vet ovario derecho de diámetro de $2,67 \mathrm{~cm}$ y el ovario izquierdo se observa con un cuerpo luteo. 
2019-04-23 — Palpación rectal con ultrasonido, se evidencia un útero con edema grado 3, diametro de $4.5 \mathrm{~cm}$, estrogenizado, el ovario derecho con un foliculo de diametro de $4,3 \mathrm{~cm}$ y el ovario izquierdo con diametro de $2,8 \mathrm{~cm}$ sin ninguna estructura.La yegua presenta celo

2019-05-08 — Palpación rectal con ultrasonido, útero con edema grado 2, diametro de $3.2 \mathrm{~cm}$, ovario derecho con un cuerpo luteo y ovario izquierdo con diametro del 2,6 cmEl cuerpo luteo es evidencia que el foliculo fue ovulado y la yegua esta ciclando normalmente

Palpación rectal con ultrasonido, se observa un edema grado 4 en el útero, con diámetro de $4.8 \mathrm{~cm}$, en el ovario derecho diámetro de $2,7 \mathrm{~cm}$ y ovario izquierdo un folículo de diámetro $4,3 \mathrm{~cm}$.La yegua es inseminada

Palpación rectal con ultrasonido, se ve un cuerpo luteo en el ovario izquierdo, .Se esperan 8 dias a partir de la evidencia de cuerpo luteo para realizar lavado uterino para obtener embrion

Se realiza el lavado uterino y se obtiene el primer embrión de la yegua.y fue transferido a una receptora.El dia 45 fue realizada la ecografia y el embrión no estaba presente, fue reabsorbido.

Palpación rectal con ultrasonido, se evidencia un folículo derecho de $4,4 \mathrm{~cm}$ de diámetro, utero con edema grado 3 con diametro $3.9 \mathrm{~cm}$, ovario izquierdo multifolicular con diametro de $3.8 \mathrm{~cm}$. derecho 4,5 cm de diametro, ovario izquierdo $4,3 \mathrm{~cm}$ de diametro, utero con edema grado 4. y diametro de $4.9 \mathrm{~cm}$.La yegua es inseminada yeguas receptoras.Se realizo ecografia via rectal el dia 60 y solo se evidencia gestacion en una sola yegua, la otra yegua presento reabsorcion embrionaria. 


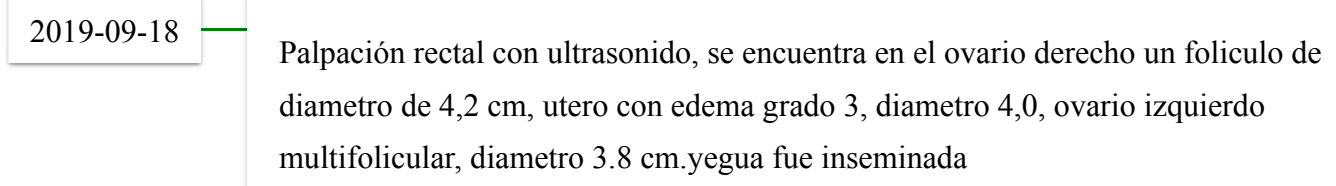

2020-01-22 - Yegua con ciclos estrales normales y fertil.Los dos embriones transferidos se encuentran en las receptoras, en espera para sus respectivos partos

\section{Narrative}

Yegua criolla colombiana de trocha pura para exposicion,fecha de nacimiento 15 de septiembre de 2014.

Yegua con problemas reproductivos, ciclos irregulares, útero pequeño y ovarios pequeños, no presenta folículos

en los ovarios y no ha sido posible obtener preñez o embriones.

Diagnosticada como yegua mosaico con dos líneas celulares:63X0/64XX,

El veterinario que la trataba realizo el siguiente protocolo:

\section{Lograr que cicle (estrógenos y progestágenos)}

2. Inseminación con semen fresco diluido de alta calidad

3. Esperar gestación directamente en la yegua.

4. Evaluar si se deja preñada o se induce una reabsorción.

No fue posible que la yegua ciclara, ni se obtuvo ninguna preñez

La madre de la yegua no presenta historia de problemas reproductivos, tiene 3 hijos por transferencia de embrión, yegua fuera de concurso en pista. 
El padre es un reproductor reconocido con múltiples crías a nivel nacional y es fuera de concurso en pista

El tratamiento realizado a la yegua, por el anterior veterinario fue protocolo con progesterona oral de concentración $4 \mathrm{mg}$, a dosis de $4.5 \mathrm{~cm}$ oral, durante 14 días, y

prostaglandina $\mathrm{F} 2$ alfa, a dosis de $1 \mathrm{~cm}$ intramuscular el ultimo día, (día 14).

En la visita inicial se identifica a la palpación rectal una yegua con inactividad ovárica y uterina.

Se realiza protocolo biorregulador a la paciente durante dos semanas y media con resultados satisfactorios. La yegua inicia ciclos estrales normales y es posible obtener embriones viables de esta yegua.

\section{Perspective}

Esta yegua de alto valor genetico, que fue descartada reproductivamente por el diagnostico de mosaicismo genetico, tuvo la oportunidad de tener una vida reproductiva normal gracias al tratamiento bioregulador instaurado que logro reestablecer su eje hipotalamo, hipofisis, ovarios, utero, sin causar consecuencias en su organismo. Su propietario esta muy contento con los resultados obtenidos hasta el dia de hoy y en palabras de el, deja saber el gran trabajo que se ha hecho consiguiendo una descendencia tan esperada, sin tener efectos secundarios en el animal y poder llevar una vida deportiva normal.

\section{$\underline{\text { Discussion }}$}

Los problemas reproductivos en las yeguas élite de raza criolla colombiana son cada vez mas frecuentes, sin encontrar una solución en los tratamientos hormonales de tipo convencional; esta problemática conlleva a que las yeguas sean descartadas reproductivamente, teniendo un alto impacto a nivel económico de los criadores del caballo criollo colombiano, dado que el valor económico de las yeguas campeonas o fuera de concurso, se obtiene a partir de su progenie, por medio de la venta de sus crías y embriones.

La paciente fue tratada con protocolos de progesterona y estrógenos, sin mostrar resultados positivos, dado que ninguno de los parámetros reproductivos de cría, gestación u obtención de embriones se lograron. A partir de un análisis citogenetico se diagnostico como yegua mosaico(infértil) reduciendo todas las posibilidades para una respuesta reproductiva activa.

El protocolo realizado con medicamentos biorreguladores ofreció resultados rápidos y efectivos, gracias a la regulación del eje hipotálamo -hipófisis-ovarios, reactivando su estado reproductivo. Los medicamentos seleccionados para la activación y bioregulacion del eje fueron Hypophysis suisinjeel, Ovarium suis injeel y Hormeel SN. Hypophysis suis-injeel y Ovarium suis injeel, actúan como estimulantes de las funciones orgánicas a nivel de ovario e hipofisis, utilizándolos en una fase de desdiferenciacion celular, ya que el problema de la yegua es a nivel de cromosomas 
. Hormeel SN, actúa como regulador de la función de todas las glándulas endocrinas que intervienen en el ciclo estral

.El Coenzyme Compositum promueve la estimulación de los sistemas enzimáticos, activador mitocondrial y formación del ATP para la demanda energética que requiere la actividad reproductiva del paciente.

Berberis-Homaccord, Nux vomica-Homaccord y Lymphomyosot, intervinieron en el tratamiento con el objetivo de detoxificar la matriz extracelular, permitiendo potencializar la comunicación celular y eliminación de toxinas. La importancia de los resultados obtenidos con la terapia bioreguladora en este paciente, radica en que sin uso de métodos invasivos ni agresivos, se logro cambiar una condición epigenética, la paciente tiene una condición reproductiva normal.

De los medicamentos anteriormente nombrados se uso una ampolla de cada producto en 1 litro de Solución salina al $0.9 \%$, se aplico 1 suero 2 veces por semana durante dos semanas y media., via intravenosa lenta.

\section{Conclusion}

Los problemas reproductivos de origen genético en yeguas, son una gran limitante a nivel de la medicina veterinaria, ya que se toman como casos para descartar, la medicina bioreguladora demuestra que no solo puede ser utilizada en enfermedades agudas, crónicas, de origen infeccioso o inflamatorio, también demuestra su potencial a nivel de problemas catalogados como citogeneticos, de los cuales no se espera una solución. Con este paciente fue posible la activación de su sistema hormonal y reproductivo obteniendo resultados positivos.

Los resultados obtenidos con este paciente, abren un camino de posibilidades, no solo a nivel de medicina veterinaria sino también para la medicina humana, en la búsqueda de nuevas herramientas para abordar casos donde no hay tratamientos o son muy pocas las posibilidades de un resultado positivo.

\section{Acknowledgements}

Mis agradecimientos a Heel Colombia por permitirme compartir estas experiencias a mas personas, para poder abrir nuevas formas de tratar pacientes, por permitirme conocer de sus productos como una nueva alternativa medica para mis pacientes sin efectos colaterales, mejorando su bienestar. Gracias por el respaldo y apoyo para poder realizar este articulo.

\section{$\underline{\text { References }}$}

1. Ley, W. B. (2004). Broodmare reproduction for the equine practitioner. Teton NewMedia.

2. McKinnon, A. O. (Ed.). (2011). Equine reproduction (2nd ed). Wiley- Blackwell.

3. Samper, J. C. , Pycock, J. F. , \& McKinnon, A. O. (2007). Current Therapy in equine reproduction. Saunders Elsevier 
4. Macfeely R. A. (1990). Domestic Animal Cytogenetic. Vol 34. Academic Press. Inc.

\section{Attachments}

\begin{tabular}{|c|c|c|c|c|c|c|c|c|}
\hline \multirow{2}{*}{\multicolumn{4}{|c|}{$\begin{array}{l}\text { FACULTAD DE MEDICINA VETERINARIA Y DE ZOOTECNIA } \\
\text { UNIDAD DE CITOGENÉTICA Y DE GENOTIPIFICACIÓN DE } \\
\text { ANIMALES DOMESTICOS }\end{array}$}} & \multicolumn{3}{|c|}{$\begin{array}{l}\text { INFORME DE RESULTADOS DE ANÁLISIS } \\
\text { DE CARIOTIPOS ANIMALES }\end{array}$} & \multirow{2}{*}{\multicolumn{2}{|c|}{$\begin{array}{l}\text { UNIVESIDAD } \\
\text { NACIONAL } \\
\text { DE COLOMBIA } \\
\text { SED E B O G OTA }\end{array}$}} \\
\hline & & & & CódIGo : B- & Yorción - 8 & คับ วด19 & & \\
\hline & & \multirow{2}{*}{\multicolumn{3}{|c|}{ EXAMEN SOLICITADO: }} & \multirow{2}{*}{\multicolumn{2}{|c|}{ CÓDIGO LC-FMVZ-UN }} & \multirow{2}{*}{\multicolumn{2}{|c|}{ FACTURA No. }} \\
\hline INFORME & EQUINO 02-19 & & & & & & & \\
\hline FECHA INFORME & $22 / 02 / 2019$ & \multicolumn{3}{|c|}{ ANÁLISIS DE CARIOTIPO } & \multicolumn{2}{|c|}{ Eq 02-19 } & \multicolumn{2}{|c|}{139566933} \\
\hline $\begin{array}{l}\text { PROPIETARIO O } \\
\text { RESPONSABLE }\end{array}$ & \multicolumn{3}{|c|}{ JUAN CAMILO ABELLO DUQUE } & DIRECCIÓN & \multicolumn{2}{|c|}{$\begin{array}{l}\text { Vereda La Balsa Sector Las } \\
\text { Juntas, Chia }\end{array}$} & $\begin{array}{l}\text { TELÉFONO } \\
\text { Y/O FAX }\end{array}$ & 3005277476 \\
\hline \multicolumn{9}{|c|}{ DESCRIPCIÓN DEL EJEMPLAR } \\
\hline ESPECIE & RAZA & COLOR & SEXO & EDAD & \multicolumn{2}{|c|}{ NOMBRE DEL EJEMPLAR } & No. REGISTRO & MICROCHIP \\
\hline Equino & $\begin{array}{c}\text { criollo } \\
\text { colombiano }\end{array}$ & castaño & hembra & 4 años & \multicolumn{2}{|c|}{ Hechizada } & 169566 & $022 * 519 * 795$ \\
\hline \multicolumn{9}{|c|}{ PROCEDENCIA: Chia, Cundinamarca } \\
\hline TIPO DE MUESTRA & \begin{tabular}{|l|} 
FECHADE \\
MUESTREO \\
\end{tabular} & $\begin{array}{l}\text { LUGAR TOMA DE } \\
\text { MUESTRA }\end{array}$ & $\begin{array}{l}\text { PROFESIC } \\
\text { TOMA LA }\end{array}$ & $\begin{array}{l}\text { NAL QUIEN } \\
\text { MUESTRA }\end{array}$ & \multicolumn{2}{|c|}{$\begin{array}{c}\text { FECHA DE SIEMBRA DE } \\
\text { MUESTRA }\end{array}$} & \multicolumn{2}{|c|}{$\begin{array}{l}\text { FECHA RECOLECCIÓN DE } \\
\text { CULTIVO }\end{array}$} \\
\hline \begin{tabular}{|l|} 
Sangre + \\
anticoagulante \\
(Heparina \\
Sódica) \\
\end{tabular} & $22 / 01 / 2019$ & Finca Betania & \multicolumn{2}{|c|}{$\begin{array}{l}\text { Dr. JUAN CAMILO } \\
\text { ABELLO }\end{array}$} & \multicolumn{2}{|c|}{$25 / 01 / 2019$} & \multicolumn{2}{|c|}{ 28/01/2019 } \\
\hline \multicolumn{9}{|c|}{ PROCESADO POR : CARLOS ARTURO SÁNCHEZ ISAZA - M.V. MP 7709 - Mg SC en Producción Animal. } \\
\hline \multirow[t]{2}{*}{ RESULTADOS: } & \multirow{2}{*}{\multicolumn{8}{|c|}{$\begin{array}{l}\text { Se analizaron al microscopio de luz un total de } 268 \text { células en metafase, se encuentraron células con } \\
\text { número cromosómico anormal } 63, \mathrm{XO}(42 \%) \text { y células con número cromosómico normal } 64, \mathrm{XX}(58 \%) \text {. }\end{array}$}} \\
\hline & & & & & & & & \\
\hline DIAGNOSTICO: & \multicolumn{8}{|c|}{ El caso corresponde a un ejemplar mosaico con dos lineas celulares: $63, \mathrm{X0} / 64, \mathrm{XX}$. } \\
\hline OBSERVACIONES: & \multicolumn{8}{|c|}{$\begin{array}{l}\text { Este tipo de anomalía cromosómica se ha reportado en yeguas asociado con problemas reproductivos. } \\
\text { Los individuos mosaicos tienen un fenotipo externo femenino más o menos normal, pero generalmente } \\
\text { tienen problemas de fertilidad y se pueden presentar ciclos estrales irregulares. }\end{array}$} \\
\hline \multicolumn{9}{|l|}{ Atentamente, } \\
\hline \multicolumn{9}{|c|}{$\begin{array}{l}\text { ORIGINAL FIRMADO } \\
\text { LIGIA MERCEDES JIMENEZ ROBAYO } \\
\text { M.V. T.P. } 7090 \text { MgSc. PhD. } \\
\text { ctor Unidad de Citogenética y de Genotipificación de Animales Domésticos } \\
\text { Profesor Asociado Facultad de Medicina Veterinaria y de Zootecnia } \\
\end{array}$} \\
\hline \multicolumn{9}{|c|}{$\begin{array}{l}\text { Universidad Nacional de Colombia. Unidad de Citogenética y de Genotipificación de Animales Domésticos. Tel. } 31656313165000 \text {, } \\
\text { ext. } 19468 \text { Bogotá D.C. Avda (Cra) } 30 \text { No- } 45-03 \text {, Ciudad Universitaria, Edificio 561-B } \\
\text { E-mail: Imjimenezr@unal.edu.co, casanchezis@unal.edu.co } \\
\text { Este informe expresa fielmente el resultado de los análisis realizados sobre la muestra recibida. El laboratorio que lo emite no se } \\
\text { responsabiliza de los perjuicios que puedan derivarse del uso inadecuado de los resultados entregados. } \\
\text { La Facultad de Medicina Veterinaria y Zootecnia no autoriza la reproducción parcial ni total de este informe. }\end{array}$} \\
\hline
\end{tabular}

Fecha:22/02/19 Examen citogenetico del paciente donde reportan un paciente con mosaicismo. 


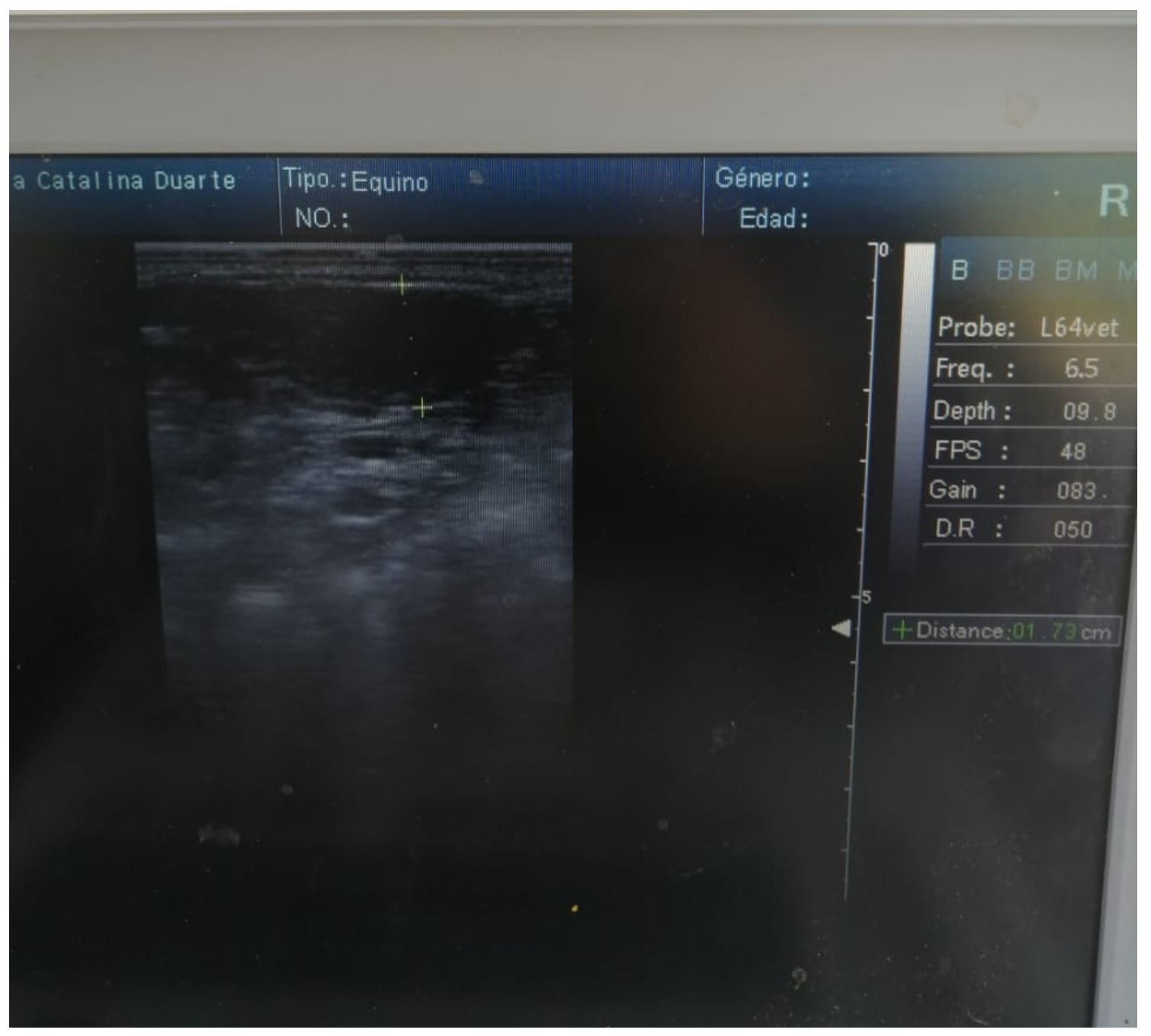

Ecografia trasrectal en paciente, se observa el utero plano de una medida de $1.73 \mathrm{~mm}$ de diametro. Antes de iniciar tratamiento bioregulador. Fecha: 20-03-2019 


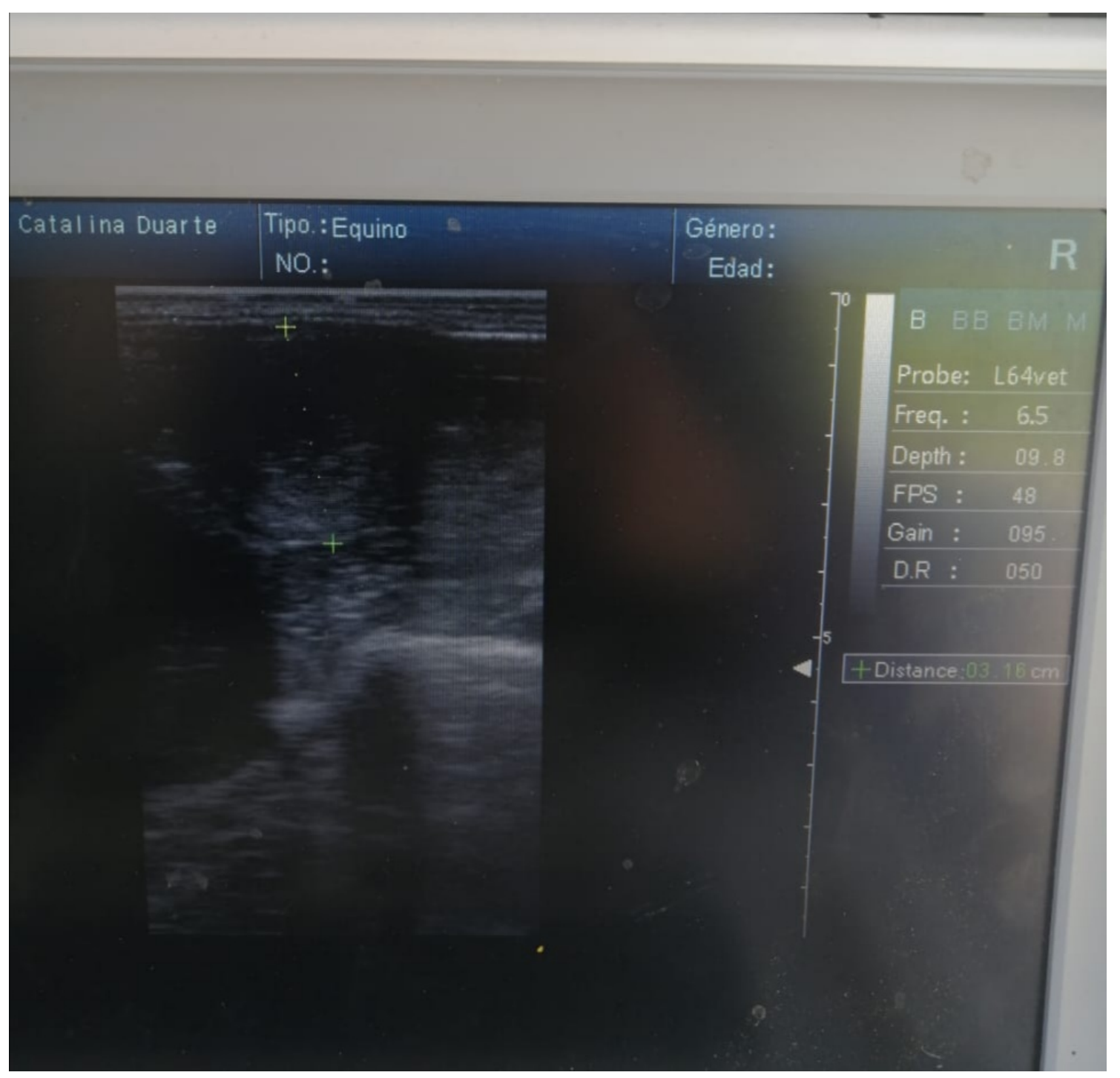

Ecografia trasrectal en paciente, se observa el utero con grado 2 de edematizacion de una medida de $3.16 \mathrm{~mm}$ de diametro. Respuesta al tratamiento bioregulador. 


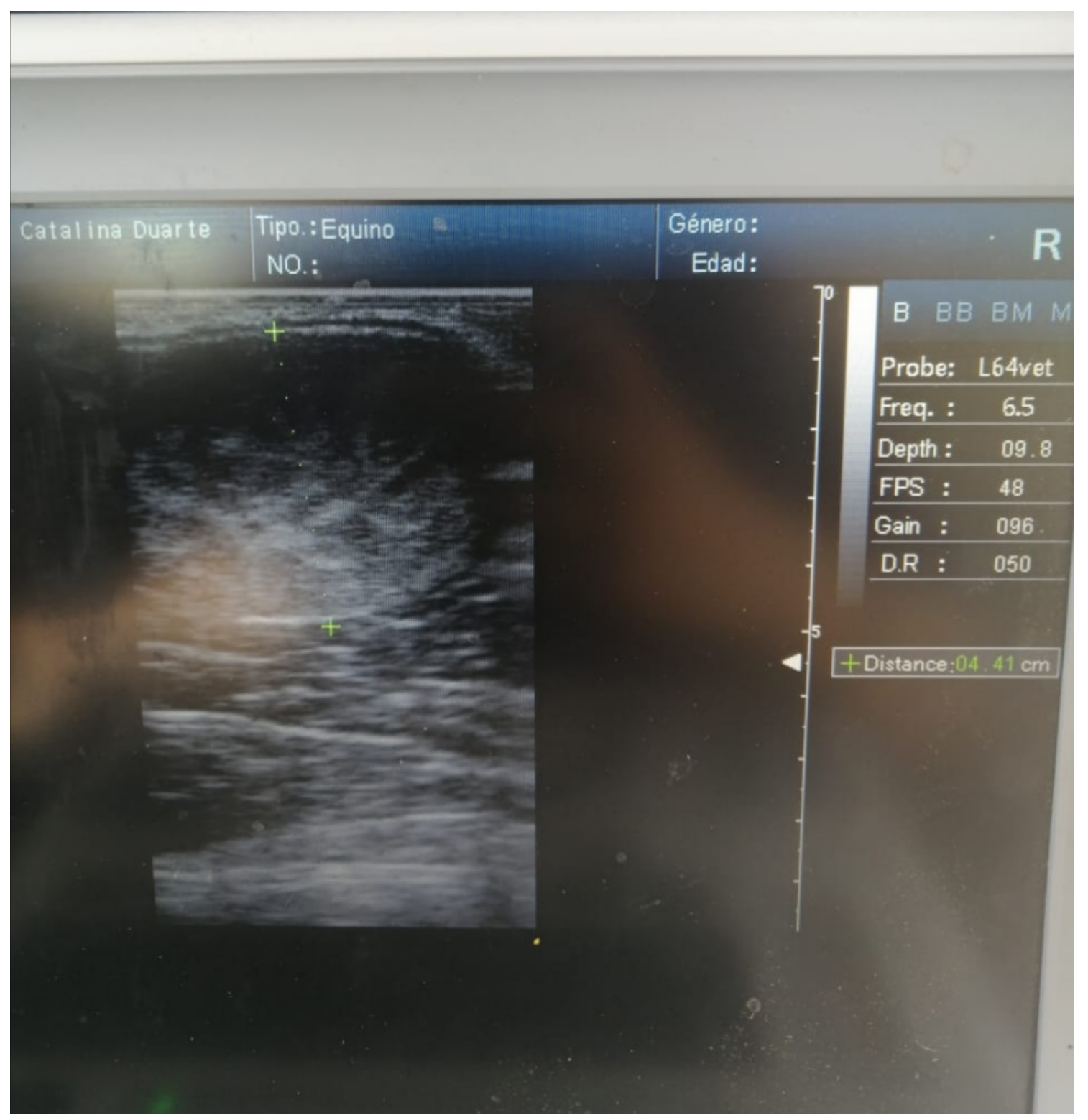

Fecha: 18/09/20 Ecografia trasrectal en paciente, se observa el utero con edema grado 3 de una medida de $4.41 \mathrm{~mm}$ de diametro. Luego de tratamiento bioregulador. 


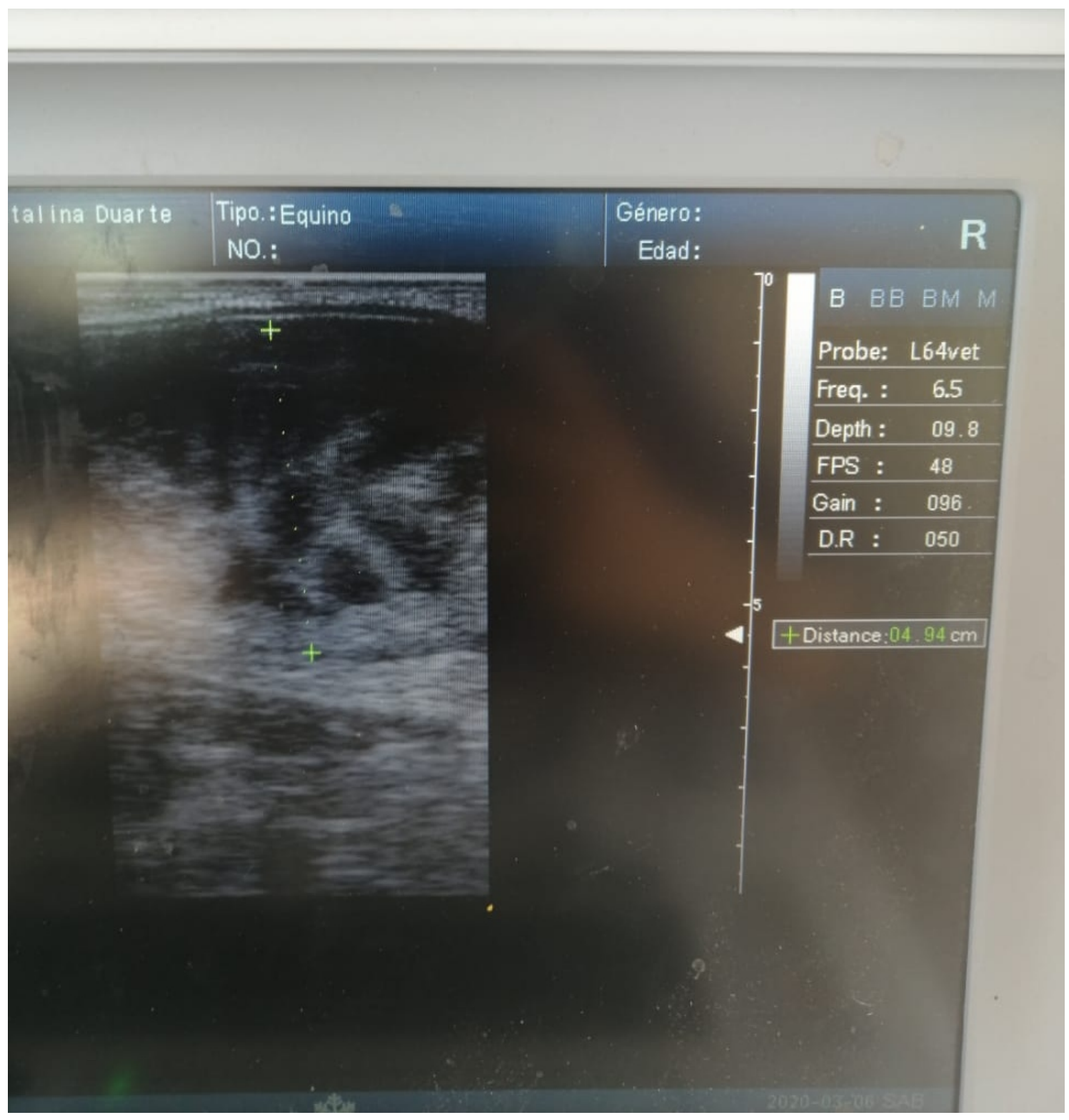

Ecografia trasrectal en paciente, se observa el utero edema 4 de una medida de $4.94 m m$ de diametro. Resultado del tratamiento bioregulador. 


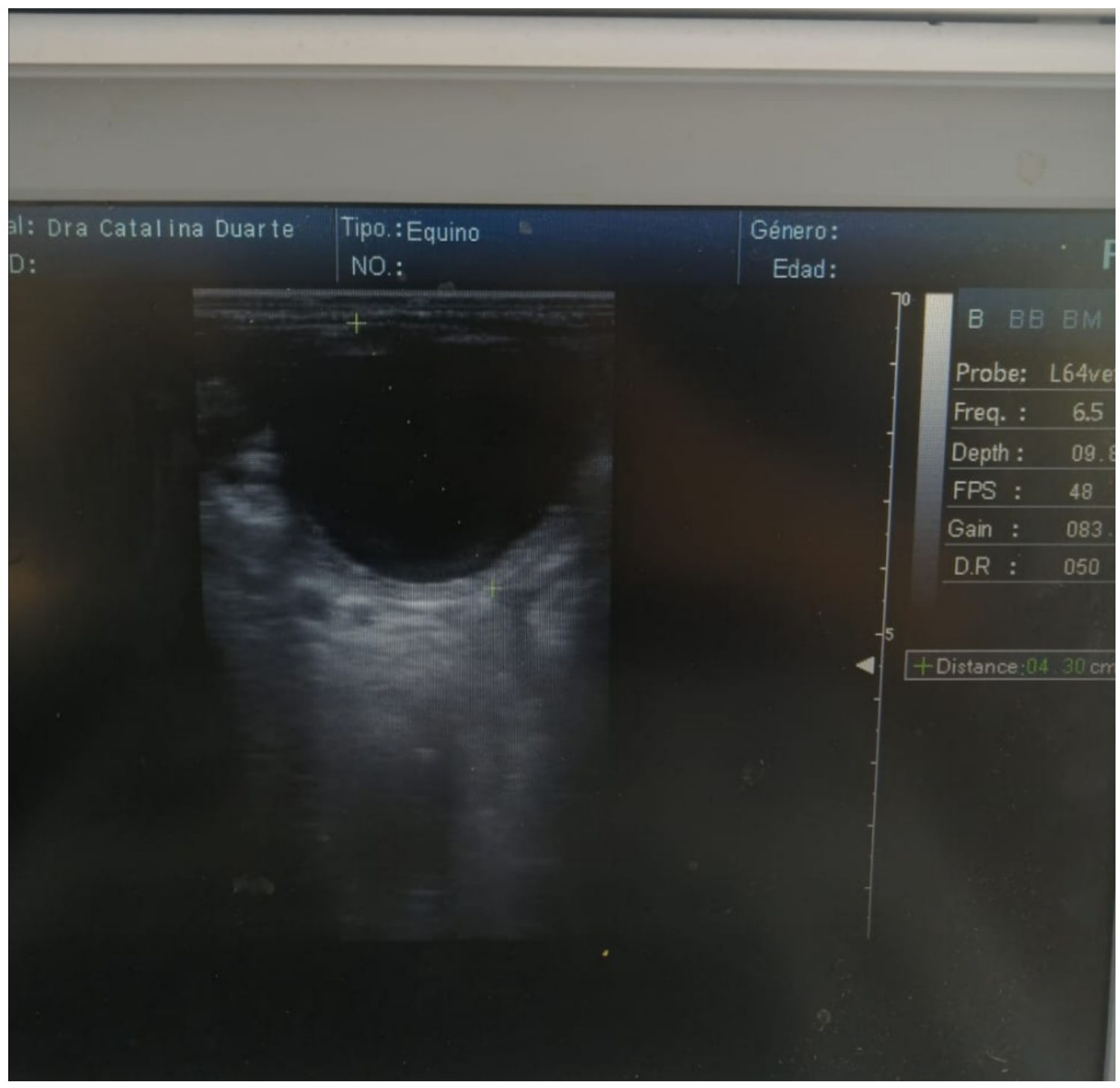

Fecha: 21/08/19 Foliculo de tamaño $4.3 \mathrm{~mm}$ listo para ovular, resultado obtenido con tratamiento bioregulador 


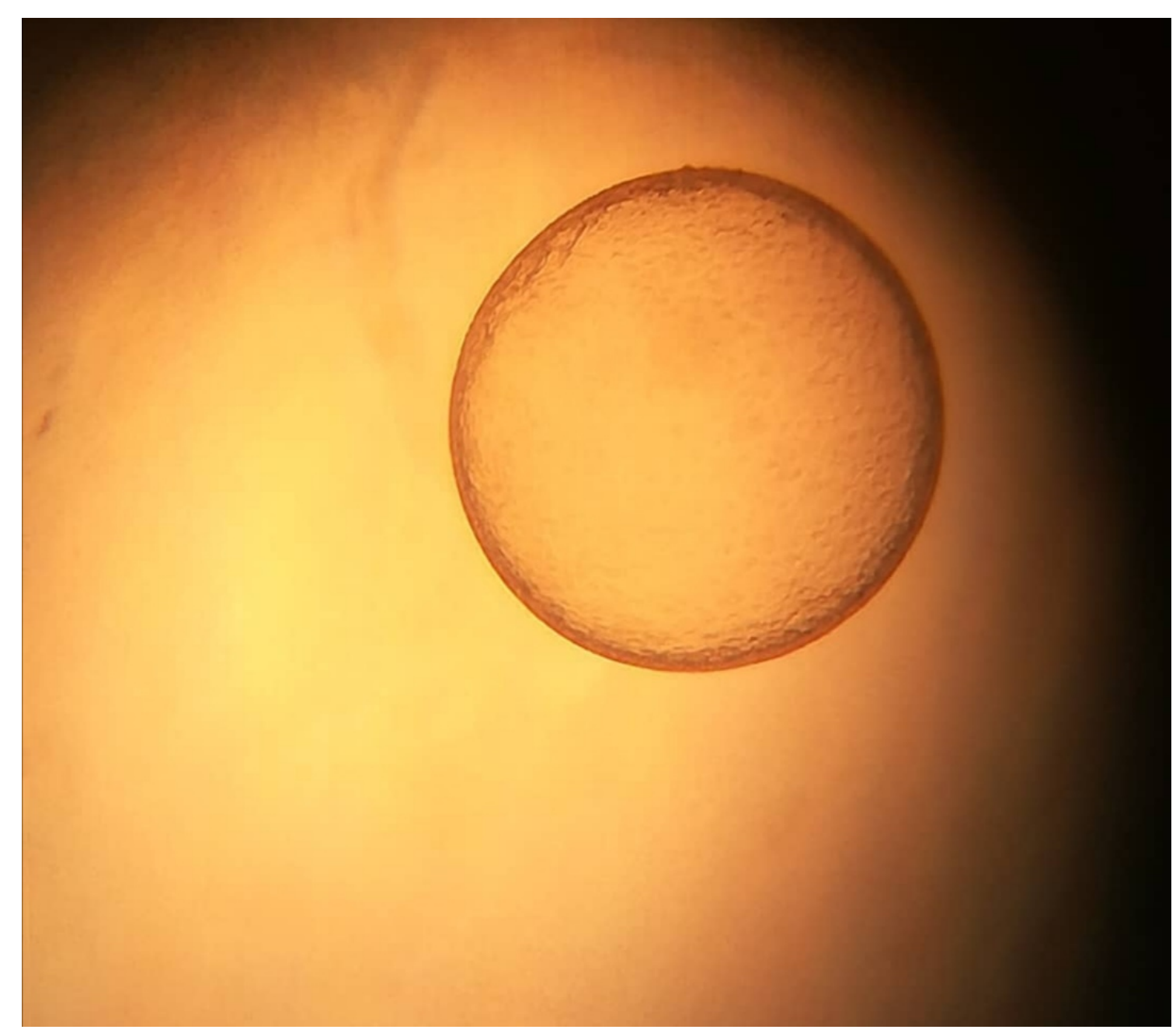

Fecha:31/05/20 Embrion de 8 dias yegua hechizada x Prodigioso 


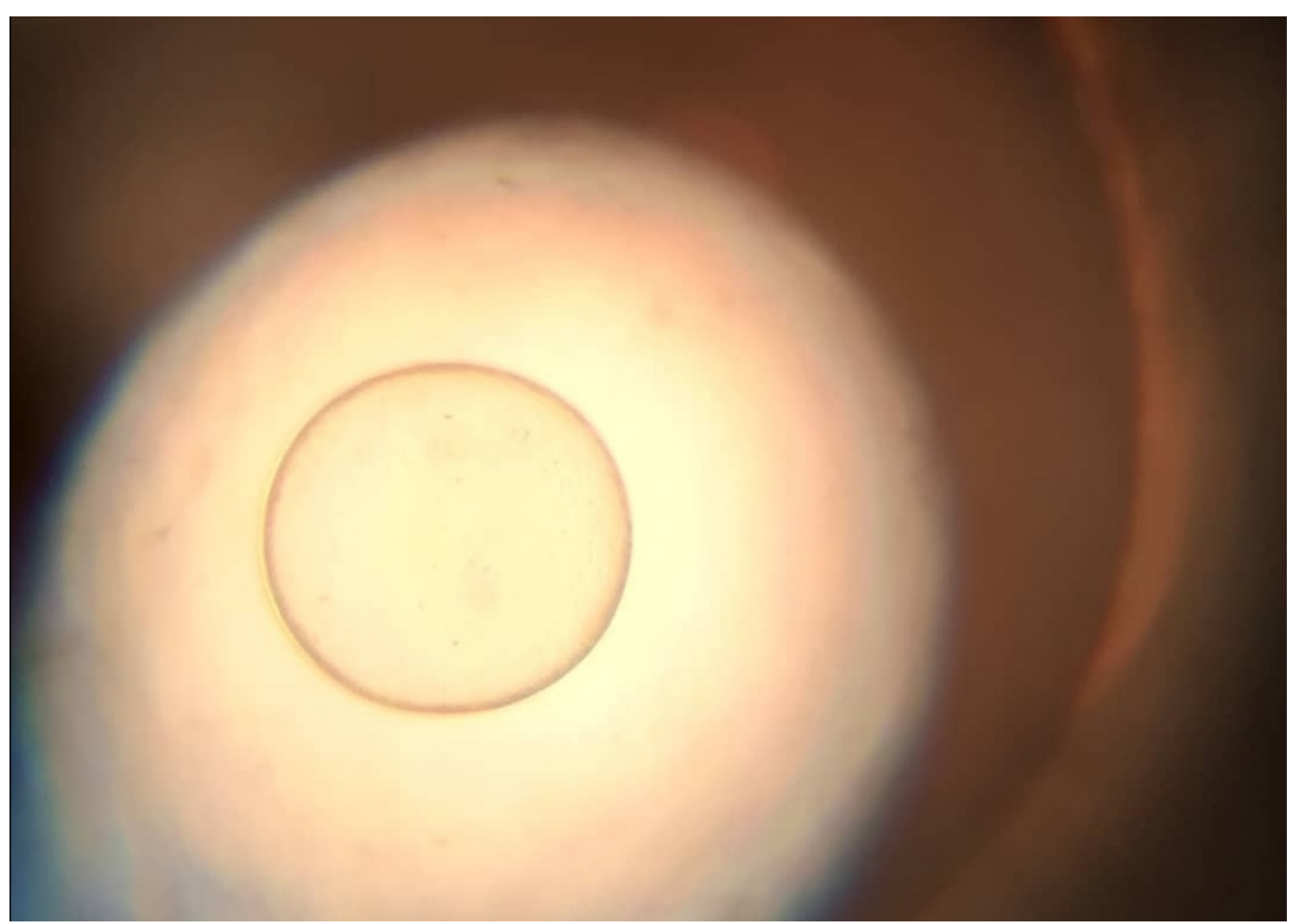

Fecha:27/09/20 Embrion de 8 dias, yegua Hechizada 


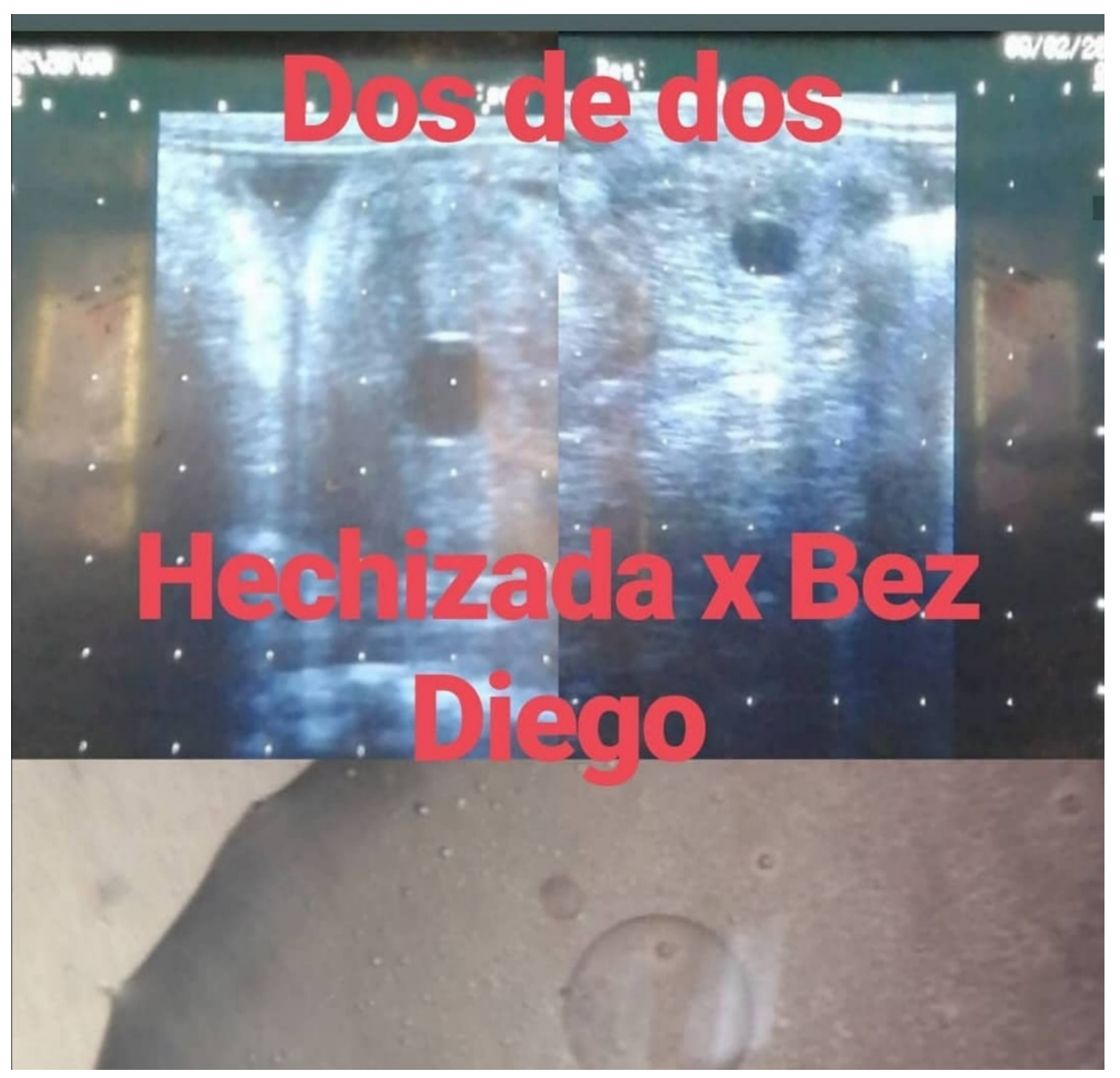

Fecha: 30/08/20 En la parte superior se observan las dos preñeces de los dos embriones obtenidos en el lavado del mes de Agosto. En la parte de abajo se observan las dos estructuras embrionarias obtenidas de la yegua antes de ser transferidos. 


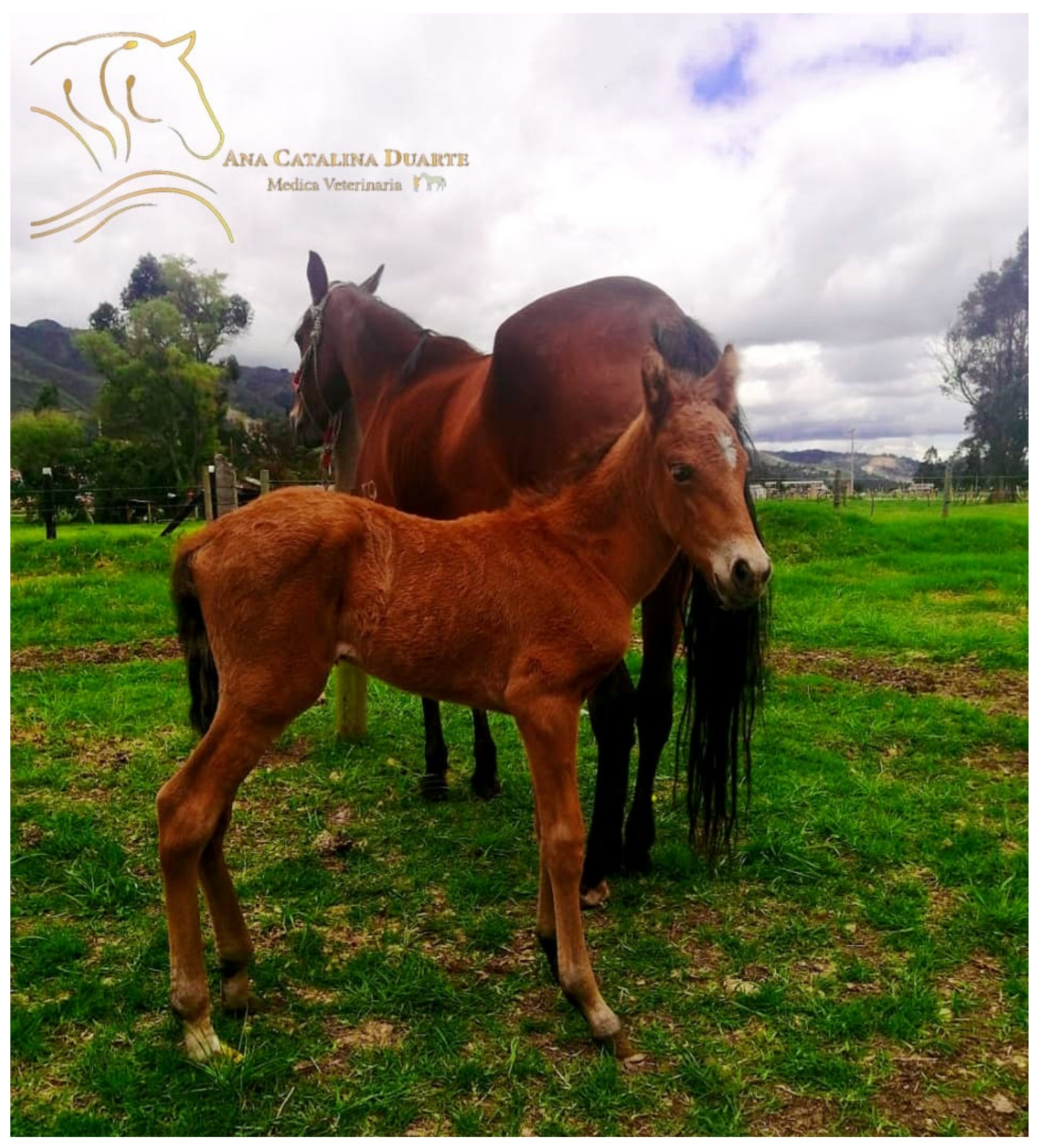

Fecha:04/08/20 Embrion y receptora 


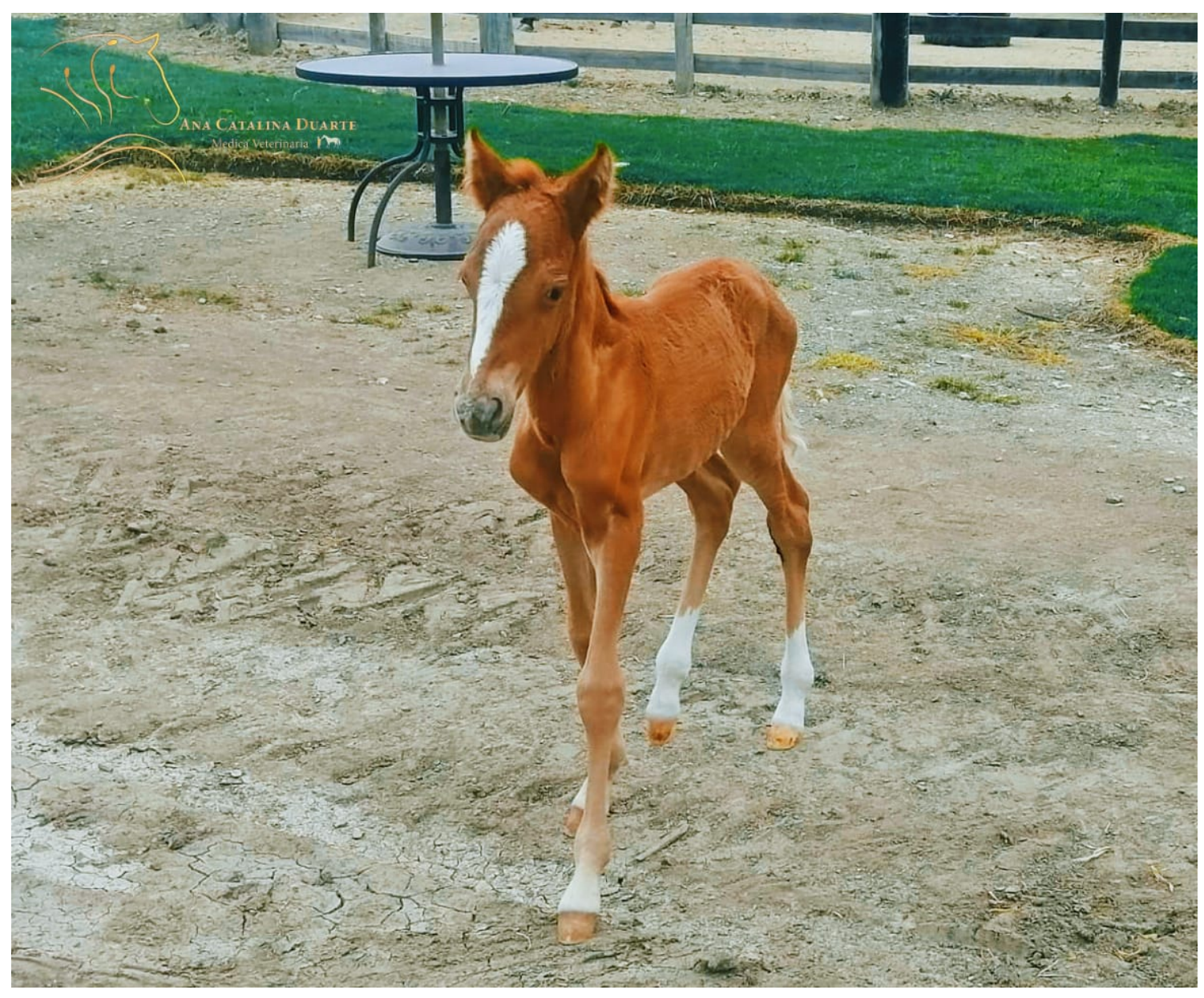

31/08/20 Embrion nacido de Hechizada x Prodigioso 


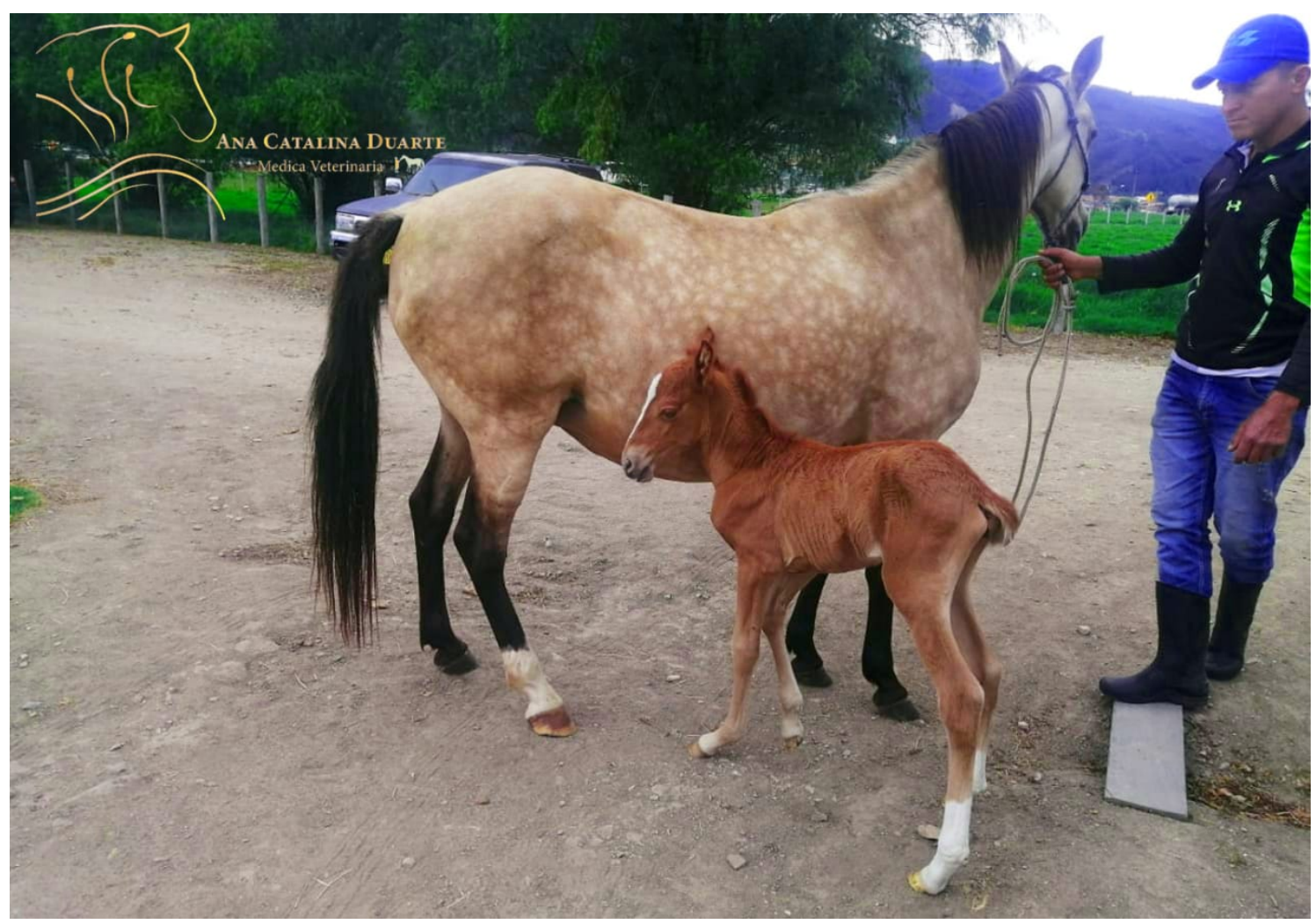

31/08/20 Embrion con la receptora, 6 horas de nacido

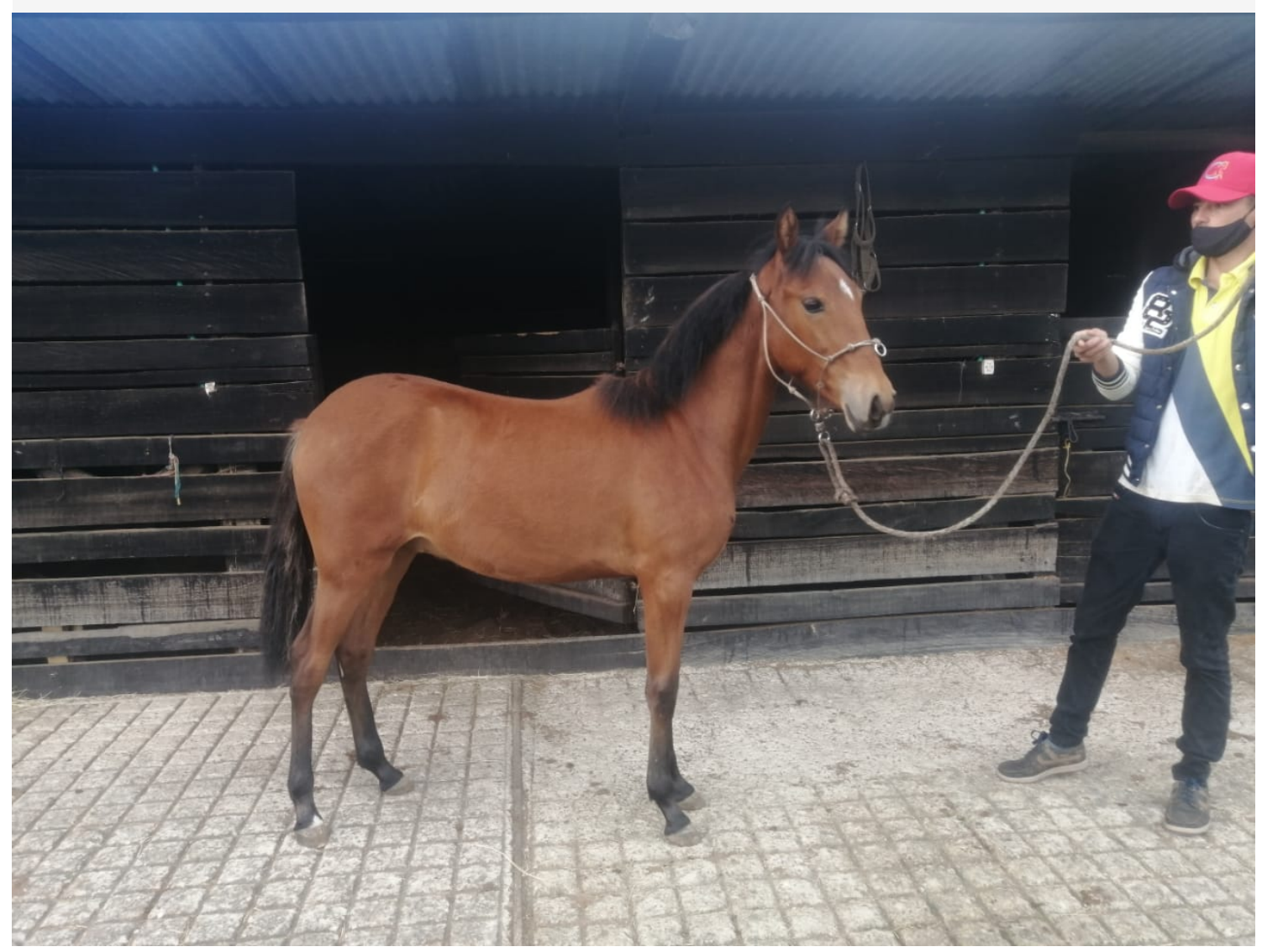

Embrion de 8 meses de edad 


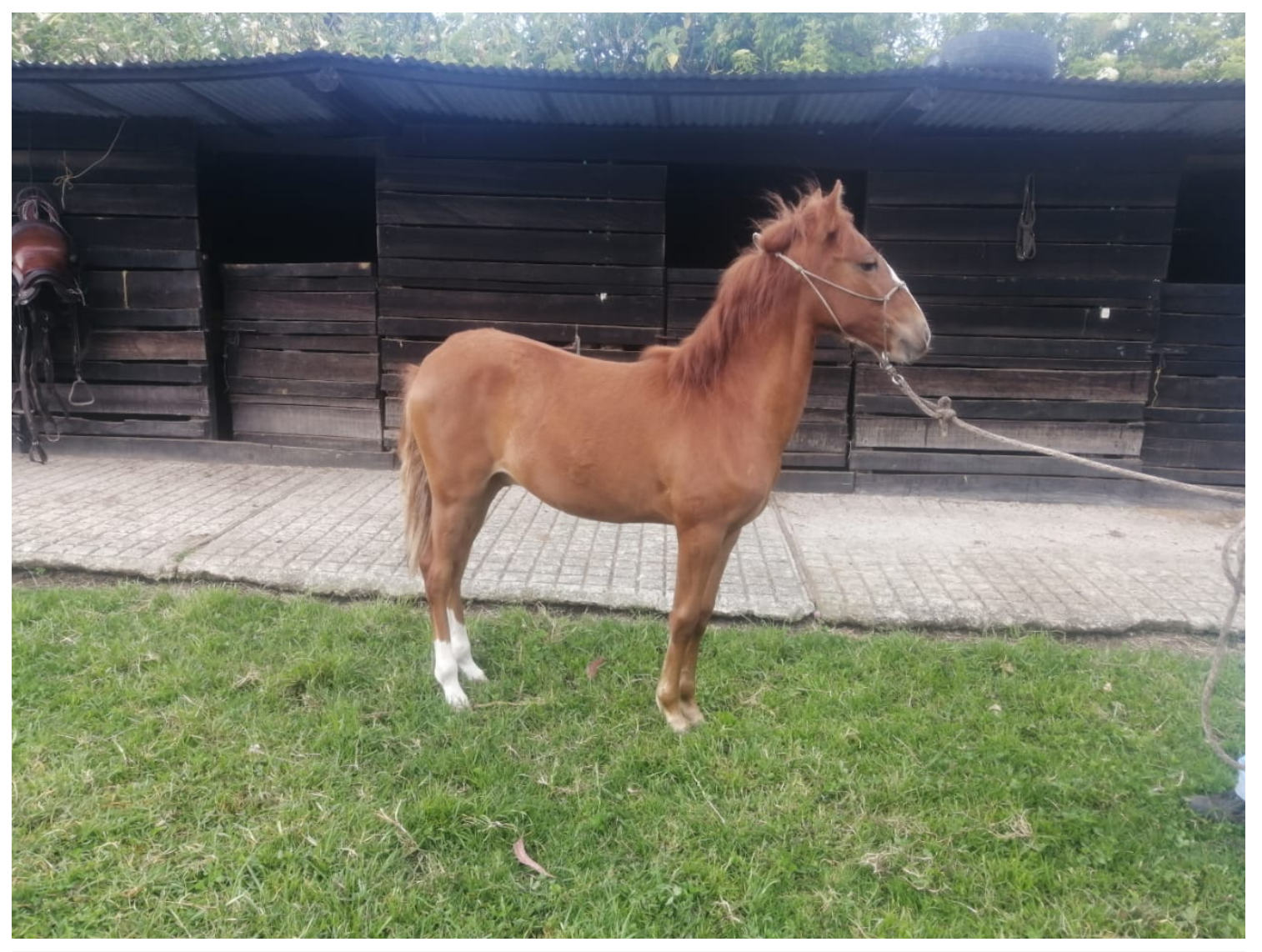

Embrion de 7 meses y medio de edad 


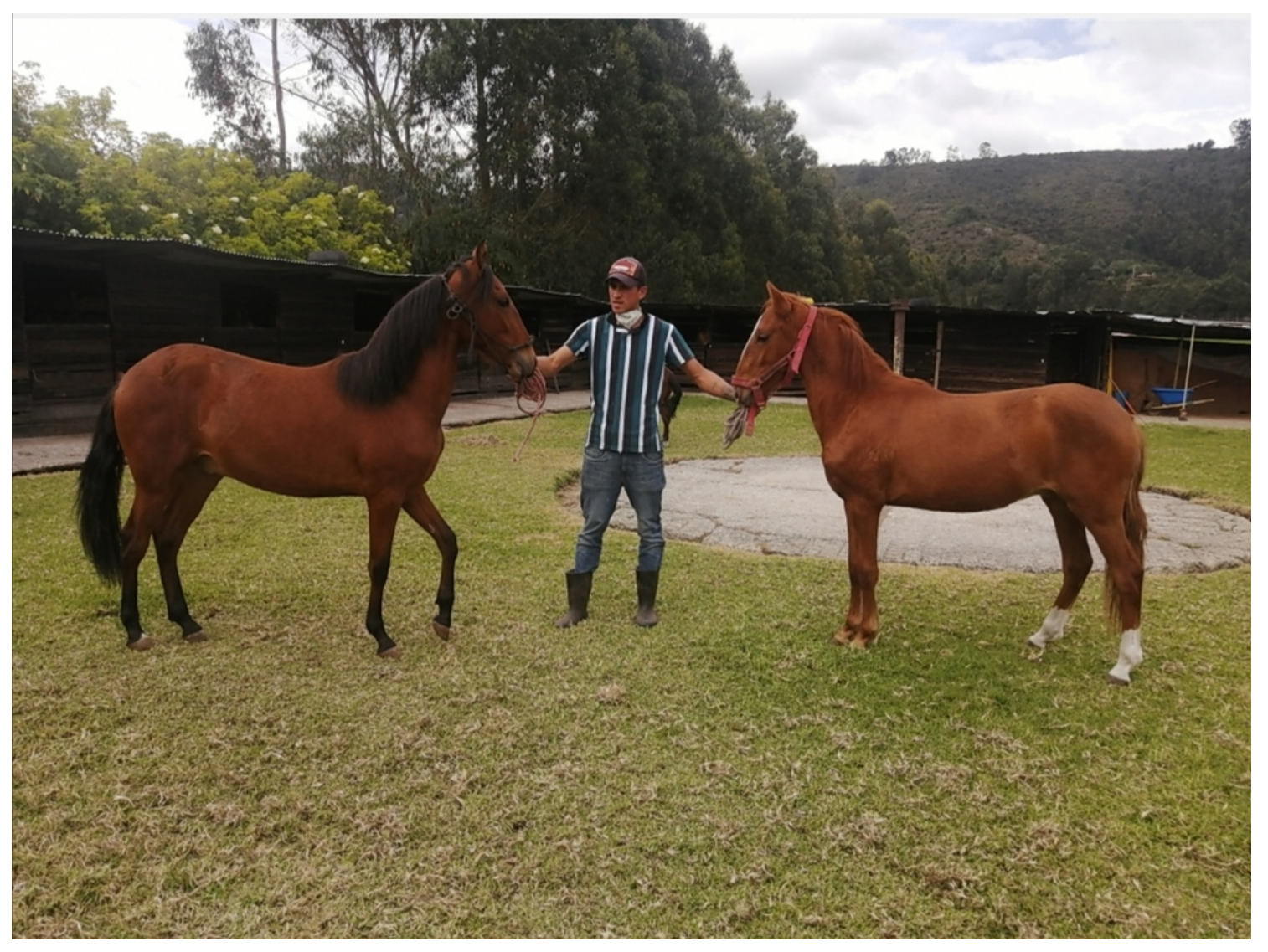

04/12/21 Dos embriones nacidos con 16 meses de edad. 